\title{
Low frequency dispersive estimates for the wave equation in higher dimensions
}

\author{
Simon Moulin
}

\begin{abstract}
We prove dispersive estimates at low frequency in dimensions $n \geq 4$ for the wave equation for a very large class of real-valued potentials, provided the zero is neither an eigenvalue nor a resonance. This class includes potentials $V \in L^{\infty}\left(\mathbf{R}^{n}\right)$ satisfying $V(x)=O\left(\langle x\rangle^{-(n+1) / 2-\epsilon}\right)$, $\epsilon>0$.
\end{abstract}

\section{Introduction and statement of results}

High frequency dispersive estimates with loss of $(n-3) / 2$ have been recently proved in [9] for the wave equation with a real-valued potential $V \in L^{\infty}\left(\mathbf{R}^{n}\right), n \geq 4$, satisfying

$$
|V(x)| \leq C\langle x\rangle^{-\delta}, \quad \forall x \in \mathbf{R}^{n},
$$

with constants $C>0, \delta>(n+1) / 2$. The problem of proving dispersive estimates at low frequency, however, left open. The purposes of the present paper is to address this problem. Such low frequency dispersive estimates for the Schrödinger group have been recently proved in [7] for a large class of real-valued potentials (not necessarily in $L^{\infty}$ ), and in particular for potentials satisfying $(1.1)$ with $\delta>(n+2) / 2$.

Denote by $G_{0}$ and $G$ the self-adjoint realizations of the operators $-\Delta$ and $-\Delta+V$ on $L^{2}\left(\mathbf{R}^{n}\right)$, respectively. It is well known that, under the condition (1.1), the absolutely continuous spectrums of the operators $G_{0}$ and $G$ coincide with the interval $[0,+\infty)$, and that $G$ has no embedded strictly positive eigenvalues nor strictly positive resonances. However, $G$ may have in general a finite number of non-positive eigenvalues and that the zero may be a resonance. We will say that the zero is a regular point for $G$ if it is neither an eigenvalue nor a resonance in the sense that the operator $1-V \Delta^{-1}$ is invertible on $L^{1}$ with a bounded inverse denoted by $T$. Let $P_{a c}$ denote the spectral projection onto the absolutely continuous spectrum of $G$. Given any $a>0$, set $\chi_{a}(\sigma)=\chi_{1}(\sigma / a)$, where $\chi_{1} \in C^{\infty}(\mathbf{R}), \chi_{1}(\sigma)=0$ for $\sigma \leq 1, \chi_{1}(\sigma)=1$ for $\sigma \geq 2$. Set $\eta_{a}=\chi\left(1-\chi_{a}\right)$, where $\chi$ denotes the characteristic function of the interval $[0,+\infty)$. Clearly, $\eta_{a}(G)+\chi_{a}(G)=P_{a c}$. As in the case of the Schrödinger group (see [7]), the dispersive estimates for the low frequency part $e^{i t \sqrt{G}} \eta_{a}(G), a>0$ small, turn out to be easier to prove when $n \geq 4$, and this can be done for a larger class of potentials. In the present paper we will do so for potentials satisfying

$$
\sup _{y \in \mathbf{R}^{n}} \int_{\mathbf{R}^{n}}\left(|x-y|^{-n+2}+|x-y|^{-(n-1) / 2}\right)|V(x)| d x \leq C<+\infty .
$$

Clearly, (1.2) is fulfilled for potentials satisfying (1.1). Our main result is the following

Theorem 1.1 Let $n \geq 4$, let $V$ satisfy (1.2) and assume that the zero is a regular point for $G$. Then, there exists a constant $a_{0}>0$ so that for every $0<a \leq a_{0}, 0<\epsilon \ll 1$, $t$, we have 
the estimates

$$
\begin{gathered}
\left\|e^{i t \sqrt{G}} G^{-(n+1) / 4} \eta_{a}(G)\right\|_{L^{1} \rightarrow L^{\infty}} \leq C\langle t\rangle^{-(n-1) / 2} \log (|t|+2), \\
\left\|e^{i t \sqrt{G}} G^{-(n+1) / 4+\epsilon} \eta_{a}(G)\right\|_{L^{1} \rightarrow L^{\infty}} \leq C_{\epsilon}\langle t\rangle^{-(n-1) / 2} .
\end{gathered}
$$

Moreover, for every $2 \leq p<+\infty$, we have the estimate

$$
\left\|e^{i t \sqrt{G}} G^{-\alpha(n+1) / 4} \eta_{a}(G)\right\|_{L^{p^{\prime} \rightarrow L^{p}}} \leq C\langle t\rangle^{-\alpha(n-1) / 2},
$$

where $1 / p+1 / p^{\prime}=1, \alpha=1-2 / p$, provided the operator $T$ is bounded on $L^{p^{\prime}}$.

Remark 1. Note that our proof of the above estimates works out in the case $n=3$, too, for potentials satysfying (1.2) as well as the condition $V \in L^{3 / 2-\epsilon}$ with some $0<\epsilon \ll 1$. In this case, however, a similar result has been already proved by D'ancona and Pierfelice [5]. In fact, in [5] the whole range of frequencies has been treated for a very large subset of Kato potentials.

Combining Theorem 1.1 with the estimates of [9], we obtain the following

Corollary 1.2 Let $n \geq 4$, let $V$ satisfy (1.1) and assume that the zero is a regular point for $G$. Then, for every $2 \leq p<+\infty, 0<\epsilon \ll 1, t \neq 0$, we have the estimates

$$
\begin{gathered}
\left\|e^{i t \sqrt{G}} G^{-(n+1) / 4}\langle G\rangle^{-(n-3) / 4-\epsilon} P_{a c}\right\|_{L^{1} \rightarrow L^{\infty}} \leq C_{\epsilon}|t|^{-(n-1) / 2} \log (|t|+2), \\
\left\|e^{i t \sqrt{G}} G^{-(n+1) / 4+\epsilon}\langle G\rangle^{-(n-3) / 4-2 \epsilon} P_{a c}\right\|_{L^{1} \rightarrow L^{\infty}} \leq C_{\epsilon}|t|^{-(n-1) / 2}, \\
\left\|e^{i t \sqrt{G}} G^{-\alpha(n+1) / 4}\langle G\rangle^{-\alpha(n-3) / 4} P_{a c}\right\|_{L^{p^{\prime} \rightarrow L^{p}}} \leq C|t|^{-\alpha(n-1) / 2},
\end{gathered}
$$

where $1 / p+1 / p^{\prime}=1, \alpha=1-2 / p$. Moreover, for every $0 \leq q \leq(n-3) / 2,2 \leq p<\frac{2(n-1-2 q)}{(n-3-2 q)}$, we have

$$
\left\|e^{i t \sqrt{G}} G^{-\alpha(n+1) / 4}\langle G\rangle^{-\alpha q / 2} P_{a c}\right\|_{L^{p^{\prime} \rightarrow L^{p}}} \leq C|t|^{-\alpha(n-1) / 2} .
$$

Note that when $n=2$ and $n=3$ similar dispersive estimates (without loss of derivatives) for the high frequency part $e^{i t \sqrt{G}} \chi_{a}(G)$ are proved in [2] for potentials satisfying (1.1) (see also [3], [5]). For higher dimensions Beals [1] proved optimal (without loss of derivatives) dispersive estimates for potentials belonging to the Schwartz class. It seems that to avoid the loss of derivatives in dimensions $n \geq 4$ one needs to impose some regularity condition on the potential. Similar phenomenon also occurs in the case of the Schrödinger equation (see [4]). Note that dispersive estimates without loss of derivatives for the Schrödinger group $e^{i t G}$ in dimensions $n \geq 4$ are proved in $[6]$ under the regularity condition $\widehat{V} \in L^{1}$. This result has been recently extended in [7] to potentials $V$ satisfying (1.1) with $\delta>n-1$ as well as $\widehat{V} \in L^{1}$.

To prove Theorem 1.1 we adapt the approach of [7] to the wave equation. It consists of proving uniform $L^{1} \rightarrow L^{\infty}$ dispersive estimates for the operator $e^{i t \sqrt{G}} \psi\left(h^{2} G\right)$, where $\psi \in C_{0}^{\infty}((0,+\infty)), h \gg 1$. To do so, we use Duhamel's formula for the wave equation (which in our case takes the form (2.12)). It turns out that when $n \geq 4$ one can absorb the remaining terms taking the parameter $h$ big enough, so one does not need anymore to work on weighted $L^{2}$ spaces (as in [9]). This allows to cover a larger class of potentials not necessarily in $L^{\infty}$. 


\section{Proof of Theorem 1.1}

Let $\psi \in C_{0}^{\infty}((0,+\infty))$. The following proposition is proved in [7] and that is why we omit the proof.

Proposition 2.1 Under the assumptions of Theorem 1.1, there exist positive constants $C, \beta$ and $h_{0}$ so that the following estimates hold

$$
\begin{gathered}
\left\|\psi\left(h^{2} G_{0}\right)\right\|_{L^{1} \rightarrow L^{1}} \leq C, \quad h>0, \\
\left\|\psi\left(h^{2} G\right)\right\|_{L^{1} \rightarrow L^{1}} \leq C, \quad h \geq h_{0}, \\
\left\|\psi\left(h^{2} G\right)-\psi\left(h^{2} G_{0}\right) T\right\|_{L^{1} \rightarrow L^{1}} \leq C h^{-\beta}, \quad h \geq h_{0},
\end{gathered}
$$

where the operator

$$
T=\left(1-V \Delta^{-1}\right)^{-1}: L^{1} \rightarrow L^{1}
$$

is bounded by assumption.

Set

$$
\Phi(t, h)=e^{i t \sqrt{G}} \psi\left(h^{2} G\right)-T^{*} e^{i t \sqrt{G_{0}}} \psi\left(h^{2} G_{0}\right) T .
$$

We will first show that Theorem 1.1 follows from the following

Proposition 2.2 Under the assumptions of Theorem 1.1, there exist positive constants $C$, $h_{0}$ and $\beta$ so that for all $h \geq h_{0}, t$, we have

$$
\|\Phi(t, h)\|_{L^{1} \rightarrow L^{\infty}} \leq C h^{-(n+1) / 2-\beta}\langle t\rangle^{-(n-1) / 2} .
$$

By interpolation between (2.5) and the trivial bound

$$
\|\Phi(t, h)\|_{L^{2} \rightarrow L^{2}} \leq C,
$$

we obtain

$$
\|\Phi(t, h)\|_{L^{p^{\prime} \rightarrow L^{p}}} \leq C h^{-\alpha(n+1) / 2-\alpha \beta}\langle t\rangle^{-\alpha(n-1) / 2},
$$

for every $2 \leq p \leq+\infty$, where $1 / p+1 / p^{\prime}=1, \alpha=1-2 / p$. Now, writing

$$
\sigma^{-\alpha(n+1) / 4} \eta_{a}(\sigma)=\int_{a^{-1}}^{\infty} \psi(\sigma \theta) \theta^{\alpha(n+1) / 4} \frac{d \theta}{\theta}, \quad \sigma>0,
$$

where $\psi(\sigma)=\sigma^{1-\alpha(n+1) / 4} \chi_{1}^{\prime}(\sigma) \in C_{0}^{\infty}((0,+\infty)$ ), and using (2.7) we get (for $2<p \leq+\infty$ )

$$
\begin{gathered}
\left\|e^{i t \sqrt{G}} G^{-\alpha(n+1) / 4} \eta_{a}(G)-T^{*} e^{i t \sqrt{G_{0}}} G_{0}^{-\alpha(n+1) / 4} \eta_{a}\left(G_{0}\right) T\right\|_{L^{p^{\prime} \rightarrow L^{p}}} \\
\leq \int_{a^{-1}}^{\infty}\|\Phi(t, \sqrt{\theta})\|_{L^{p^{\prime} \rightarrow L^{p}}} \theta^{-1+\alpha(n+1) / 4} d \theta \\
\leq C\langle t\rangle^{-\alpha(n-1) / 2} \int_{a^{-1}}^{\infty} \theta^{-1-\alpha \beta / 2} d \theta \leq C\langle t\rangle^{-\alpha(n-1) / 2},
\end{gathered}
$$

provided $a$ is taken small enough. The estimate (1.5) follows from (2.8) and the fact that it holds for $G_{0}$ (see [8]). Clearly, (1.3) follows from (2.8) with $p=+\infty$ and the estimate (A.1) in the appendix. In the same way we get

$$
\left\|e^{i t \sqrt{G}} G^{-(n+1) / 4+\epsilon} \eta_{a}(G)-T^{*} e^{i t \sqrt{G_{0}}} G_{0}^{-(n+1) / 4+\epsilon} \eta_{a}\left(G_{0}\right) T\right\|_{L^{1} \rightarrow L^{\infty}} \leq C\langle t\rangle^{-(n-1) / 2},
$$

which together with the estimate (A.2) in the appendix imply (1.4).

Proof of Proposition 2.2. We will derive (2.5) from the following 
Proposition 2.3 Under the assumptions of Theorem 1.1, there exist positive constants $C$, $h_{0}$ and $\beta$ so that we have, for $\forall f \in L^{1}$,

$$
\begin{gathered}
\left\|e^{i t \sqrt{G_{0}}} \psi\left(h^{2} G_{0}\right) f\right\|_{L^{\infty}} \leq C h^{-(n+1) / 2}\langle t\rangle^{-(n-1) / 2}\|f\|_{L^{1}}, \quad h \geq 1, \forall t, \\
\int_{-\infty}^{\infty}\left\|V e^{i t \sqrt{G_{0}}} \psi\left(h^{2} G_{0}\right) f\right\|_{L^{1}} d t \leq C h^{-(n-1) / 2}\|f\|_{L^{1}}, \quad h>0, \\
\int_{-\infty}^{\infty}\left\|V e^{i t \sqrt{G}} \psi\left(h^{2} G\right) f\right\|_{L^{1}} d t \leq C h^{-1-\beta}\|f\|_{L^{1}}, \quad h \geq h_{0} .
\end{gathered}
$$

We use Duhamel's formula

$$
e^{i t \sqrt{G}}=e^{i t \sqrt{G_{0}}}+i \frac{\sin \left(t \sqrt{G_{0}}\right)}{\sqrt{G_{0}}}\left(\sqrt{G}-\sqrt{G_{0}}\right)-\int_{0}^{t} \frac{\sin \left((t-\tau) \sqrt{G_{0}}\right)}{\sqrt{G_{0}}} V e^{i \tau \sqrt{G}} d \tau
$$

to get the identity

$$
\Phi(t ; h)=\sum_{j=1}^{2} \Phi_{j}(t ; h)
$$

where

$$
\begin{gathered}
\Phi_{1}(t ; h)=\left(\psi_{1}\left(h^{2} G\right)-T^{*} \psi_{1}\left(h^{2} G_{0}\right)\right) e^{i t \sqrt{G}} \psi\left(h^{2} G\right) \\
+T^{*} \psi_{1}\left(h^{2} G_{0}\right) e^{i t \sqrt{G_{0}}}\left(\psi\left(h^{2} G\right)-\psi\left(h^{2} G_{0}\right) T\right) \\
-i T^{*} \psi_{1}\left(h^{2} G_{0}\right) \sin \left(t \sqrt{G_{0}}\right)\left(\psi\left(h^{2} G\right)-\psi\left(h^{2} G_{0}\right) T\right) \\
+i T^{*} \widetilde{\psi}_{1}\left(h^{2} G_{0}\right) \sin \left(t \sqrt{G_{0}}\right)\left(\widetilde{\psi}\left(h^{2} G\right)-\widetilde{\psi}\left(h^{2} G_{0}\right) T\right), \\
\Phi_{2}(t ; h)=-h \int_{0}^{t} T^{*} \widetilde{\psi}_{1}\left(h^{2} G_{0}\right) \sin \left((t-\tau) \sqrt{G_{0}}\right) V e^{i \tau \sqrt{G}} \psi\left(h^{2} G\right) d \tau,
\end{gathered}
$$

where $\psi_{1} \in C_{0}^{\infty}((0,+\infty)), \psi_{1}=1$ on $\operatorname{supp} \psi, \widetilde{\psi}(\sigma)=\sigma^{1 / 2} \psi(\sigma), \widetilde{\psi}_{1}(\sigma)=\sigma^{-1 / 2} \psi_{1}(\sigma)$. Let $t>0$. By Propositions 2.1 and 2.3, we have

$$
\begin{gathered}
\quad\left\|\Phi_{1}(t ; h) f\right\|_{L^{\infty}} \leq C h^{-(n+1) / 2-\beta}\langle t\rangle^{-(n-1) / 2}\|f\|_{L^{1}}+C h^{-\beta}\|\Phi(t ; h) f\|_{L^{\infty}}, \\
\leq h \int_{0}^{t / 2}\langle t-\tau\rangle^{(n-1) / 2}\left\|\sin \left((t-\tau) \sqrt{G_{0}}\right) \widetilde{\psi}_{1}\left(h^{2} G_{0}\right) T g\right\|_{L^{\infty}}\left\|V e^{i \tau \sqrt{G}} \psi\left(h^{2} G\right) f\right\|_{L^{1}} d \tau \\
\quad+h \int_{t / 2}^{t}\left\|V \sin \left((t-\tau) \sqrt{G_{0}}\right) \widetilde{\psi}_{1}\left(h^{2} G_{0}\right) T g\right\|_{L^{1}}\langle\tau\rangle^{(n-1) / 2}\left\|e^{i \tau \sqrt{G}} \psi\left(h^{2} G\right) f\right\|_{L^{\infty}} d \tau \\
\leq C h^{-(n-1) / 2}\|g\|_{L^{1}} \int_{-\infty}^{\infty}\left\|V e^{i \tau \sqrt{G}} \psi\left(h^{2} G\right) f\right\|_{L^{1}} d \tau \\
\left.+h \sup _{t / 2 \leq \tau \leq t}\langle\tau\rangle\right\rangle^{(n-1) / 2}\left\|e^{i \tau \sqrt{G}} \psi\left(h^{2} G\right) f\right\|_{L^{\infty}} \int_{-\infty}^{\infty}\left\|V \sin \left((t-\tau) \sqrt{G_{0}}\right) \widetilde{\psi}_{1}\left(h^{2} G_{0}\right) T g\right\|_{L^{1}} d \tau \\
\leq C h^{-(n+1) / 2-\beta}\|g\|_{L^{1}}\|f\|_{L^{1}}+C h^{-\beta}\|g\|_{L^{1}} \sup _{t / 2 \leq \tau \leq t}\langle\tau\rangle
\end{gathered}
$$

which clearly implies

$$
\langle t\rangle^{(n-1) / 2}\left\|\Phi_{2}(t ; h) f\right\|_{L^{\infty}} \leq C h^{-(n+1) / 2-\beta}\|f\|_{L^{1}}
$$




$$
+C h^{-\beta} \sup _{t / 2 \leq \tau \leq t}\langle\tau\rangle^{(n-1) / 2}\left\|e^{i \tau \sqrt{G}} \psi\left(h^{2} G\right) f\right\|_{L^{\infty}} .
$$

By (2.12)-(2.14), we conclude

$$
\begin{gathered}
\langle t\rangle^{(n-1) / 2}\|\Phi(t ; h) f\|_{L^{\infty}} \leq C h^{-(n+1) / 2-\beta}\|f\|_{L^{1}}+C h^{-\beta}\langle t\rangle^{(n-1) / 2}\|\Phi(t ; h) f\|_{L^{\infty}} \\
+C h^{-\beta} \sup _{t / 2 \leq \tau \leq t}\langle\tau\rangle^{(n-1) / 2}\|\Phi(\tau ; h) f\|_{L^{\infty}} .
\end{gathered}
$$

Taking $h$ big enough we can absorb the second and the third terms in the RHS of (2.15), thus obtaining (2.5). Clearly, the case of $t<0$ can be treated in the same way.

\section{Proof of Proposition 2.3.}

We will make use of the fact that the kernel of the operator $e^{i t \sqrt{G_{0}}} \psi\left(h^{2} G_{0}\right)$ is of the form $K_{h}(|x-y|, t)$, where

$$
K_{h}(\sigma, t)=\frac{\sigma^{-2 \nu}}{(2 \pi)^{\nu+1}} \int_{0}^{\infty} e^{i t \lambda} \mathcal{J}_{\nu}(\sigma \lambda) \psi\left(h^{2} \lambda^{2}\right) \lambda d \lambda=h^{-n} K_{1}\left(\sigma h^{-1}, t h^{-1}\right),
$$

where $\mathcal{J}_{\nu}(z)=z^{\nu} J_{\nu}(z), J_{\nu}(z)=\left(H_{\nu}^{+}(z)+H_{\nu}^{-}(z)\right) / 2$ is the Bessel function of order $\nu=$ $(n-2) / 2$. It is shown in [9] (Section 2) that $K_{h}$ satisfies the estimates (for all $\sigma, t>0$, $h \geq 1)$

$$
\begin{gathered}
\left|K_{1}(\sigma, t)\right| \leq C\langle t\rangle^{-s}\langle\sigma\rangle^{s-(n-1) / 2}, \quad \forall s \geq 0 \\
\left|K_{h}(\sigma, t)\right| \leq C h^{-(n+1) / 2}\langle t\rangle^{-s} \sigma^{s-(n-1) / 2}, \quad 0 \leq s \leq(n-1) / 2 .
\end{gathered}
$$

Clearly, (2.9) follows from (3.3) with $s=(n-1) / 2$. It is not hard to see that $(2.10)$ follows from (1.2) and the following

Lemma 3.1 For all $\sigma, h>0,0 \leq s \leq(n-1) / 2$, we have

$$
\int_{-\infty}^{\infty}|t|^{s}\left|K_{h}(\sigma, t)\right| d t \leq C h^{-(n-1) / 2} \sigma^{s-(n-1) / 2}
$$

Proof. In view of (3.1), it suffices to show (3.4) with $h=1$. When $0<\sigma \leq 1$, this follows from (3.2). Let now $\sigma \geq 1$. We will use the fact that the function $\mathcal{J}_{\nu}$ can be decomposed as $\mathcal{J}_{\nu}(z)=e^{i z} b_{\nu}^{+}(z)+e^{-i z} b_{\nu}^{-}(z)$, where $b_{\nu}^{ \pm}(z)$ are symbols of order $(n-3) / 2$ for $z \geq 1$. Then, we can decompose the function $K_{1}$ as $K_{1}^{+}+K_{1}^{-}$, where $K_{1}^{ \pm}$are defined by replacing in the definition of $K_{1}$ the function $\mathcal{J}_{\nu}(\sigma \lambda)$ by $e^{ \pm i \sigma \lambda} b_{\nu}^{ \pm}(\sigma \lambda)$. Integrating by parts, we get

$$
\left|K_{1}^{ \pm}(\sigma, t)\right| \leq C_{m} \sigma^{-(n-1) / 2}|t \pm \sigma|^{-m}
$$

for every integer $m \geq 0$. By (3.5),

$$
\begin{gathered}
\int_{-\infty}^{\infty}|t|^{s}\left|K_{1}^{ \pm}(\sigma, t)\right| d t \leq \sigma^{s} \int_{-\infty}^{\infty}\left|K_{1}^{ \pm}(\sigma, t)\right| d t+\int_{-\infty}^{\infty}|t \pm \sigma|^{s}\left|K_{1}^{ \pm}(\sigma, t)\right| d t \\
\leq C_{m} \sigma^{s-(n-1) / 2} \int_{-\infty}^{\infty}|t \pm \sigma|^{-m} d t+C_{m} \sigma^{-(n-1) / 2} \int_{-\infty}^{\infty}|t \pm \sigma|^{-m+s} d t \leq C \sigma^{s-(n-1) / 2}
\end{gathered}
$$

which clearly implies (3.4) in this case.

To prove (2.11) we will use the formula

$$
e^{i t \sqrt{G}} \psi\left(h^{2} G\right)=(i \pi h)^{-1} \int_{0}^{\infty} e^{i t \lambda} \varphi_{h}(\lambda)\left(R^{+}(\lambda)-R^{-}(\lambda)\right) d \lambda,
$$


where $\varphi_{h}(\lambda)=\varphi_{1}(h \lambda), \varphi_{1}(\lambda)=\lambda \psi\left(\lambda^{2}\right)$, and $R^{ \pm}(\lambda)=\left(G-\lambda^{2} \pm i 0\right)^{-1}$ satisfy the identity

$$
R^{ \pm}(\lambda)\left(1+V R_{0}^{ \pm}(\lambda)\right)=R_{0}^{ \pm}(\lambda)
$$

Here $R_{0}^{ \pm}(\lambda)$ denote the outgoing and incoming free resolvents with kernels given in terms of the Hankel functions, $H_{\nu}^{ \pm}$, of order $\nu=(n-2) / 2$ by the formula

$$
\left[R_{0}^{ \pm}(\lambda)\right](x, y)= \pm i 4^{-1}(2 \pi)^{-\nu}|x-y|^{-n+2} \mathcal{H}_{\nu}^{ \pm}(\lambda|x-y|),
$$

where $\mathcal{H}_{\nu}^{ \pm}(z)=z^{\nu} H_{\nu}^{ \pm}(z)$ satisfy

$$
\begin{gathered}
\left|\partial_{z}^{j} \mathcal{H}_{\nu}^{ \pm}(z)\right| \leq C\langle z\rangle^{(n-3) / 2}, \quad \forall z>0, j=0,1, \\
\left|\mathcal{H}_{\nu}^{ \pm}(z)-\mathcal{H}_{\nu}^{ \pm}(0)\right| \leq C z^{1 / 2}\langle z\rangle^{(n-4) / 2}, \quad \forall z>0 .
\end{gathered}
$$

It follows easily from these bounds and (1.2) that

$$
\begin{gathered}
\left\|V R_{0}^{ \pm}(\lambda)\right\|_{L^{1} \rightarrow L^{1}} \leq C, \quad 0<\lambda \leq 1, \\
\left\|V R_{0}^{ \pm}(\lambda)-V R_{0}^{ \pm}(0)\right\|_{L^{1} \rightarrow L^{1}} \leq C \lambda^{1 / 2}, \quad 0<\lambda \leq 1 .
\end{gathered}
$$

Since $1+V R_{0}^{ \pm}(0)=1-V \Delta^{-1}$ is invertible on $L^{1}$ by assumption with a bounded inverse denoted by $T$, it follows from (3.10) that there exists a constant $\lambda_{0}>0$ so that the operator $1+V R_{0}^{ \pm}(\lambda)$ is invertible on $L^{1}$ for $0<\lambda \leq \lambda_{0}$. In view of (3.8), we have

$$
\begin{gathered}
\sum_{ \pm} \pm V R^{ \pm}(\lambda)=-\sum_{ \pm} \pm\left(1+V R_{0}^{ \pm}(\lambda)\right)^{-1}=-\sum_{ \pm} \pm T\left(1+\left(V R_{0}^{ \pm}(\lambda)-V R_{0}^{ \pm}(0)\right) T\right)^{-1} \\
=\sum_{ \pm} \pm T\left(V R_{0}^{ \pm}(\lambda)-V R_{0}^{ \pm}(0)\right) T\left(1+\left(V R_{0}^{ \pm}(\lambda)-V R_{0}^{ \pm}(0)\right) T\right)^{-1}
\end{gathered}
$$

By (3.7) and (3.11),

$$
V e^{i t \sqrt{G}} \psi\left(h^{2} G\right)=(i \pi h)^{-1} \sum_{ \pm} \pm \int_{-\infty}^{\infty} T V P_{h}^{ \pm}(t-\tau) U_{h}^{ \pm}(\tau) d \tau
$$

where

$$
\begin{gathered}
P_{h}^{ \pm}(t)=\int_{0}^{\infty} e^{i t \lambda} \widetilde{\varphi}_{h}(\lambda)\left(R_{0}^{ \pm}(\lambda)-R_{0}^{ \pm}(0)\right) d \lambda, \\
U_{h}^{ \pm}(t)=\int_{0}^{\infty} e^{i t \lambda} \varphi_{h}(\lambda) T\left(1+\left(V R_{0}^{ \pm}(\lambda)-V R_{0}^{ \pm}(0)\right) T\right)^{-1} d \lambda,
\end{gathered}
$$

where $\widetilde{\varphi}_{h}(\lambda)=\widetilde{\varphi}_{1}(h \lambda), \widetilde{\varphi}_{1} \in C_{0}^{\infty}((0,+\infty))$ is such that $\widetilde{\varphi}_{1}=1$ on $\operatorname{supp} \varphi_{1}$. The kernel of the operator $P_{h}^{ \pm}(t)$ is of the form $A_{h}^{ \pm}(|x-y|, t)$, where

$$
\left.A_{h}^{ \pm}(\sigma, t)= \pm i 4^{-1}(2 \pi)^{-\nu} \sigma^{-n+2} \int_{0}^{\infty} e^{i t \lambda} \widetilde{\varphi}_{h}(\lambda)\left(\mathcal{H}_{\nu}^{ \pm}(\sigma \lambda)\right)-\mathcal{H}_{\nu}^{ \pm}(0)\right) d \lambda=h^{1-n} A_{1}^{ \pm}(\sigma / h, t / h) .
$$

Lemma 3.2 For all $\sigma>0, h \geq 1$, we have

$$
\int_{-\infty}^{\infty}\left|A_{h}^{ \pm}(\sigma, t)\right| d t \leq C h^{-1 / 2}\left(\sigma^{-n+5 / 2}+\sigma^{-(n-1) / 2}\right) .
$$


Proof. In view of (3.13), it suffices to prove (3.14) with $h=1$. Consider first the case $0<\sigma \leq 1$. Using the inequality

$$
\|\widehat{f}\|_{L^{1}} \leq C \sum_{j=0}^{1} \sup _{\lambda}\langle\lambda\rangle\left|\partial_{\lambda}^{j} f(\lambda)\right|
$$

we get

$$
\sigma^{n-2} \int_{-\infty}^{\infty}\left|A_{1}^{ \pm}(\sigma, t)\right| d t \leq C \sup _{\lambda \in \operatorname{supp} \widetilde{\varphi}_{1}}\left(\left|\mathcal{H}_{\nu}^{ \pm}(\sigma \lambda)-\mathcal{H}_{\nu}^{ \pm}(0)\right|+\sigma\left|\partial_{\lambda} \mathcal{H}_{\nu}^{ \pm}(\sigma \lambda)\right|\right) \leq C \sigma^{1 / 2}
$$

which is the desired bound. Let now $\sigma \geq 1$. We have

$$
A_{1}^{ \pm}(\sigma, t)=K_{1}^{ \pm}(\sigma, t)+c^{ \pm} \sigma^{-n+2} \int_{0}^{\infty} e^{i t \lambda} \widetilde{\varphi}_{1}(\lambda) d \lambda
$$

where $c^{ \pm}$are constants and $K_{1}^{ \pm}$are as in the proof of Lemma 3.1. Hence, in this case, (3.14) (with $h=1$ ) follows from (3.6) (with $s=0$ ).

By (3.12), (3.14) and (1.2), we have

$$
\begin{gathered}
\int_{-\infty}^{\infty}\left\|V e^{i t \sqrt{G}} \psi\left(h^{2} G\right) f\right\|_{L^{1}} d t \leq C h^{-1} \sum_{ \pm} \int_{-\infty}^{\infty} \int_{-\infty}^{\infty}\left\|V P_{h}^{ \pm}(t-\tau) U_{h}^{ \pm}(\tau) f\right\|_{L^{1}} d \tau d t \\
\leq C h^{-1} \sum_{ \pm} \int_{-\infty}^{\infty} \int_{-\infty}^{\infty} \int_{\mathbf{R}^{n}} \int_{\mathbf{R}^{n}}|V(x)|\left|A_{h}^{ \pm}(|x-y|, t-\tau)\right|\left|U_{h}^{ \pm}(\tau) f(y)\right| d x d y d \tau d t \\
\leq C h^{-1} \sum_{ \pm} \int_{\mathbf{R}^{n}} \int_{\mathbf{R}^{n}}|V(x)|\left(\int_{-\infty}^{\infty}\left|A_{h}^{ \pm}(|x-y|, \tau)\right| d \tau\right)\left(\int_{-\infty}^{\infty}\left|U_{h}^{ \pm}(\tau) f(y)\right| d \tau\right) d x d y \\
\leq C h^{-3 / 2} \sum_{ \pm} \int_{\mathbf{R}^{n}} \int_{\mathbf{R}^{n}}|V(x)|\left(|x-y|^{-n+5 / 2}+|x-y|^{-(n-1) / 2}\right) \int_{-\infty}^{\infty}\left|U_{h}^{ \pm}(\tau) f(y)\right| d \tau d x d y \\
\leq C h^{-3 / 2} \sum_{ \pm} \int_{\mathbf{R}^{n}} \int_{-\infty}^{\infty}\left|U_{h}^{ \pm}(\tau) f(y)\right| d \tau d y .
\end{gathered}
$$

Thus, (2.11) follows from (3.15) and the following

Lemma 3.3 There exists a constant $h_{0}>0$ so that for $h \geq h_{0}$ we have

$$
\int_{\mathbf{R}^{n}} \int_{-\infty}^{\infty}\left|U_{h}^{ \pm}(t) f(x)\right| d t d x \leq C\|f\|_{L^{1}}
$$

Proof. Using the identity

$$
\begin{gathered}
T\left(1+\left(V R_{0}^{ \pm}(\lambda)-V R_{0}^{ \pm}(0)\right) T\right)^{-1} \\
=T-T\left(V R_{0}^{ \pm}(\lambda)-V R_{0}^{ \pm}(0)\right) T\left(1+\left(V R_{0}^{ \pm}(\lambda)-V R_{0}^{ \pm}(0)\right) T\right)^{-1},
\end{gathered}
$$

we obtain

$$
U_{h}^{ \pm}(t)=T \widehat{\varphi}_{h}(t)-\int_{-\infty}^{\infty} T V P_{h}^{ \pm}(t-\tau) U_{h}^{ \pm}(\tau) d \tau
$$

Since

$$
\int_{-\infty}^{\infty}\left|\widehat{\varphi}_{h}(t)\right| d t=h^{-1} \int_{-\infty}^{\infty}\left|\widehat{\varphi}_{1}(t / h)\right| d t=\int_{-\infty}^{\infty}\left|\widehat{\varphi}_{1}(t)\right| d t
$$


as above, we have

$$
\begin{gathered}
\int_{\mathbf{R}^{n}} \int_{-\infty}^{\infty}\left|U_{h}^{ \pm}(t) f(x)\right| d t d x \leq C\|f\|_{L^{1}} \int_{-\infty}^{\infty}\left|\widehat{\varphi}_{h}(t)\right| d t \\
+C \int_{-\infty}^{\infty} \int_{-\infty}^{\infty} \int_{\mathbf{R}^{n}} \int_{\mathbf{R}^{n}}|V(x)|\left|A_{h}^{ \pm}(|x-y|, t-\tau)\right|\left|U_{h}^{ \pm}(\tau) f(y)\right| d x d y d \tau d t \\
\leq C\|f\|_{L^{1}}+C h^{-1 / 2} \int_{\mathbf{R}^{n}} \int_{-\infty}^{\infty}\left|U_{h}^{ \pm}(\tau) f(y)\right| d \tau d y,
\end{gathered}
$$

which implies (3.16) provided $h$ is taken big enough.

\section{A Appendix}

The following low frequency dispersive estimates for the free wave group are more or less known, but we will give a proof for the sake of completeness. We have the following

Proposition A.1 Let $n \geq 3$. Then for every $0<\epsilon \ll 1$, $t$, we have the estimates

$$
\begin{gathered}
\left\|e^{i t \sqrt{G_{0}}} G_{0}^{-(n+1) / 4} \eta_{a}\left(G_{0}\right)\right\|_{L^{1} \rightarrow L^{\infty}} \leq C\langle t\rangle^{-(n-1) / 2} \log (|t|+2), \\
\left\|e^{i t \sqrt{G_{0}}} G_{0}^{-(n+1) / 4+\epsilon} \eta_{a}\left(G_{0}\right)\right\|_{L^{1} \rightarrow L^{\infty}} \leq C_{\epsilon}\langle t\rangle^{-(n-1) / 2} .
\end{gathered}
$$

Proof. The kernel of the operator in the LHS of (A.1) is of the form $K(|x-y|, t)$, where

$$
K(\sigma, t)=c_{n} \sigma^{-n+2} \int_{0}^{\infty} e^{i t \lambda} \lambda^{1-(n+1) / 2} \eta_{a}\left(\lambda^{2}\right) \mathcal{J}_{\nu}(\sigma \lambda) d \lambda
$$

When $|t| \leq 2$, using that $\mathcal{J}_{\nu}(z)=O\left(z^{n-2}\right), \forall z>0$, we have $|K(\sigma, t)| \leq$ Const, which implies (A.1) in this case. In what follows we will suppose $|t| \geq 2$. Let $\phi \in C_{0}^{\infty}(\mathbf{R}), \phi(\mu)=1$ for $|\mu| \leq 1, \phi(\mu)=0$ for $|\mu| \geq 2$. We write $K=K_{1}+K_{2}$, where

$$
\begin{gathered}
K_{1}(\sigma, t)=c_{n} \sigma^{-n+2} \int_{0}^{\infty} e^{i t \lambda} \lambda^{1-(n+1) / 2} \eta_{a}\left(\lambda^{2}\right)\left(\phi \mathcal{J}_{\nu}\right)(\sigma \lambda) d \lambda, \\
K_{2}(\sigma, t)=c_{n} \sigma^{-n+2} \int_{0}^{\infty} e^{i t \lambda} \lambda^{1-(n+1) / 2} \eta_{a}\left(\lambda^{2}\right)\left((1-\phi) \mathcal{J}_{\nu}\right)(\sigma \lambda) d \lambda .
\end{gathered}
$$

Since $\left((1-\phi) \mathcal{J}_{\nu}\right)(z)=O\left(z^{(n-3) / 2}\right), \forall z>0$, we have

$$
\left|K_{2}(\sigma, t)\right| \leq C \sigma^{-(n-1) / 2} \int_{\sigma^{-1}}^{C o n s t} \lambda^{-1} d \lambda \leq C \sigma^{-(n-1) / 2} \log \langle\sigma\rangle .
$$

It follows from (A.3) that for $|t| / 2 \leq \sigma \leq 2|t|$, we have

$$
\left|K_{2}(\sigma, t)\right| \leq C|t|^{-(n-1) / 2} \log |t| \text {. }
$$

Let now $\sigma \notin[|t| / 2,2|t|]$. We write $K_{2}$ as $K_{2}^{+}+K_{2}^{-}$, where

$$
K_{2}^{ \pm}(\sigma, t)=c_{n} \sigma^{-n+2} \int_{0}^{\infty} e^{i(t \pm \sigma) \lambda} \lambda^{1-(n+1) / 2} \eta_{a}\left(\lambda^{2}\right)\left((1-\phi) b_{\nu}^{ \pm}\right)(\sigma \lambda) d \lambda,
$$

with functions $b_{\nu}^{ \pm}$satisfying

$$
\left|\partial_{z}^{j} b_{\nu}^{ \pm}(z)\right| \leq C_{j} z^{(n-3) / 2-j}, \quad \forall j \geq 0, z \geq 1 .
$$


Integrating by parts $m \geq 1$ times we get

$$
\begin{gathered}
\left|K_{2}^{ \pm}(\sigma, t)\right| \leq C \sigma^{-n+2}|t \pm \sigma|^{-m} \int_{0}^{\infty} \sum_{j=0}^{m} \sigma^{m-j}\left|\partial_{\lambda}^{j}\left(\lambda^{1-(n+1) / 2} \eta_{a}\left(\lambda^{2}\right)\right)\right|\left|\left(\partial_{\lambda}^{m-j}(1-\phi) b_{\nu}^{ \pm}\right)(\sigma \lambda)\right| d \lambda \\
\leq C \sigma^{-n+2}|t \pm \sigma|^{-m} \int_{\sigma^{-1}}^{C o n s t} \sum_{j=0}^{m} \sigma^{m-j} \lambda^{1-(n+1) / 2-j}(\sigma \lambda)^{(n-3) / 2-(m-j)} d \lambda \\
\leq C \sigma^{-(n-1) / 2}|t \pm \sigma|^{-m} \int_{\sigma^{-1}}^{C o n s t} \lambda^{-1-m} d \lambda \leq C \sigma^{m-(n-1) / 2}|t \pm \sigma|^{-m} \int_{1}^{\infty} \mu^{-1-m} d \mu \\
\leq C \sigma^{m-(n-1) / 2}|t \pm \sigma|^{-m} \leq C_{m} \sigma^{m-(n-1) / 2}|t|^{-m}
\end{gathered}
$$

since $|t \pm \sigma| \geq|t| / 2$ in this case, for all integers $m \geq 1$, and hence for all real $m \geq 1$. Taking $m=(n-1) / 2$ in $($ A.5) we get

$$
\left|K_{2}(\sigma, t)\right| \leq C|t|^{-(n-1) / 2}, \quad \text { if } \quad \sigma \notin[|t| / 2,2|t|] .
$$

To deal with $K_{1}$ we will use that $\left(\phi \mathcal{J}_{\nu}\right)(z)=z^{n-2} g(z)$ with a function $g \in C_{0}^{\infty}(\mathbf{R})$. We write

$$
K_{1}(\sigma, t)=c_{n} \int_{0}^{\infty} e^{i t \lambda} \lambda^{(n-3) / 2} \eta_{a}\left(\lambda^{2}\right) g(\sigma \lambda) d \lambda .
$$

Lemma A.2 For every $k \geq 1$, we have

$$
\left|\int_{0}^{\infty} e^{i t \lambda} \lambda^{k-1} \eta_{a}\left(\lambda^{2}\right) g(\sigma \lambda) d \lambda\right| \leq C_{k}|t|^{-k}
$$

with a constant $C_{k}>0$ indpendent of $t$ and $\sigma$.

Proof. If $k \geq 1$ is an integer, we integrate by parts $k$ times to get

$$
\begin{gathered}
\left|\int_{0}^{\infty} e^{i t \lambda} \lambda^{k-1} \eta_{a}\left(\lambda^{2}\right) g(\sigma \lambda) d \lambda\right| \\
\leq|t|^{-k} \int_{0}^{\infty}\left|\partial_{\lambda}^{k}\left(\lambda^{k-1} \eta_{a}\left(\lambda^{2}\right) g(\sigma \lambda)\right)\right| d \lambda+|t|^{-k}\left|\partial_{\lambda}^{k-1}\left(\lambda^{k-1} \eta_{a}\left(\lambda^{2}\right) g(\sigma \lambda)\right)\right|{ }_{\lambda=0} \mid \\
\leq|t|^{-k} \int_{0}^{\infty} \sum_{j=1}^{k} \sigma^{j} \lambda^{j-1}\left|\left(\partial_{\lambda}^{j} g\right)(\sigma \lambda)\right| d \lambda+|t|^{-k} \int_{0}^{\infty}\left|\eta_{a}^{\prime}\left(\lambda^{2}\right)\right||g(\sigma \lambda)| d \lambda+|t|^{-k}|g(0)| \\
\leq\left.|t|^{-k} \sum_{j=1}^{k} \int_{0}^{\infty}(\sigma \lambda)^{j-1}\left(\partial_{\lambda}^{j} g\right)(\sigma \lambda)|d(\sigma \lambda)+| t\right|^{-k} \int_{0}^{\infty}\left|\eta_{a}^{\prime}\left(\lambda^{2}\right)\right| d \lambda+|t|^{-k}|g(0)| \leq C_{k}|t|^{-k} .
\end{gathered}
$$

For all real $k \geq 1,(\mathrm{~A} .7)$ follows easily by complex interpolation.

Applying (A.7) with $k=(n-1) / 2$ we get

$$
\left|K_{1}(\sigma, t)\right| \leq C|t|^{-(n-1) / 2}
$$

Now (A.1) follows from (A.4), (A.6) and (A.8).

To prove (A.2) observe that the function

$$
\widetilde{K}_{2}(\sigma, t)=c_{n} \sigma^{-n+2} \int_{0}^{\infty} e^{i t \lambda} \lambda^{1+2 \epsilon-(n+1) / 2} \eta_{a}\left(\lambda^{2}\right)\left((1-\phi) \mathcal{J}_{\nu}\right)(\sigma \lambda) d \lambda
$$


satisfies the bound

$$
\left|\widetilde{K}_{2}(\sigma, t)\right| \leq C \sigma^{-(n-1) / 2} \int_{\sigma^{-1}}^{C o n s t} \lambda^{-1+2 \epsilon} d \lambda \leq C_{\epsilon} \sigma^{-(n-1) / 2} .
$$

Hence, for $|t| / 2 \leq \sigma \leq 2|t|$, we have

$$
\left|\widetilde{K}_{2}(\sigma, t)\right| \leq C|t|^{-(n-1) / 2} .
$$

The rest of the proof is exactly as above.

\section{References}

[1] M. BEals, Optimal $L^{\infty}$ decay estimates for solutions to the wave equation with a potential, Commun. Partial Diff. Equations 19 (1994), 1319-1369.

[2] F. Cardoso, C. Cuevas and G. Vodev, Dispersive estimates of solutions to the wave equation with a potential in dimensions two and three, Serdica Math. J. 31 (2005), 263-278.

[3] V. Georgiev and N. Visciglia, Decay estimates for the wave equation with potential, Commun. Partial Diff. Equations 28 (2003), 1325-1369.

[4] M. Goldberg and M. Visan, A conterexample to dispersive estimates for Schrödinger operators in higher dimensions, Commun. Math. Phys. 266 (2006), 211-238.

[5] P. D'ancona And V. Pierfelice, On the wave equation with a large rough potential, J. Funct. Analysis 227 (2005), 30-77.

[6] J.-L. Journé, A. Sofer And C. Sogge, Decay estimates for Schrödinger operators, Commun. Pure Appl. Math. 44 (1991), 573-604.

[7] S. Moulin and G. Vodev, Low frequency dispersive estimates for the Schrödinger group in higher dimensions, Asymptot. Anal., to appear.

[8] R. Strichartz, Convolutions with kernels having singularities on a sphere, Trans. A.M.S. 148 (1970), 461-471.

[9] G. Vodev, Dispersive estimates of solutions to the wave equation with a potential in dimensions $n \geq 4$, Commun. Partial Diff. Equations 31 (2006), 1709-1733.

Université de Nantes, Département de Mathématiques, UMR 6629 du CNRS, 2, rue de la Houssinière, BP 92208, 44332 Nantes Cedex 03, France

e-mail: simon.moulin@math.univ-nantes.fr 